\title{
On negative imaginary synthesis via solutions to Riccati equations*
}

DOI:

10.23919/ECC.2018.8550517

\section{Document Version}

Accepted author manuscript

Link to publication record in Manchester Research Explorer

\section{Citation for published version (APA):}

Salcan Reyes, G., \& Lanzon, A. (2018). On negative imaginary synthesis via solutions to Riccati equations*. In European Control Conference https://doi.org/10.23919/ECC.2018.8550517

\section{Published in: \\ European Control Conference}

\section{Citing this paper}

Please note that where the full-text provided on Manchester Research Explorer is the Author Accepted Manuscript or Proof version this may differ from the final Published version. If citing, it is advised that you check and use the publisher's definitive version.

\section{General rights}

Copyright and moral rights for the publications made accessible in the Research Explorer are retained by the authors and/or other copyright owners and it is a condition of accessing publications that users recognise and abide by the legal requirements associated with these rights.

\section{Takedown policy}

If you believe that this document breaches copyright please refer to the University of Manchester's Takedown Procedures [http://man.ac.uk/04Y6Bo] or contact uml.scholarlycommunications@manchester.ac.uk providing relevant details, so we can investigate your claim.

\section{OPEN ACCESS}




\title{
On negative imaginary synthesis via solutions to Riccati equations*
}

\author{
Gabriela Salcán-Reyes ${ }^{1,2}$ and Alexander Lanzon ${ }^{1}$
}

\begin{abstract}
This paper tackles the problem of finding conditions for the existence of an output feedback controller such that the closed-loop system becomes strictly negative imaginary (SNI) and internally stable. In order to achieve this objective, we derive a set of necessary and sufficient conditions to check the SNI property of systems with a non necessarily minimal state-space realization. Then, we propose a set of sufficient conditions for the controller synthesis problem and we also provide a formula for such controller.
\end{abstract}

\section{INTRODUCTION}

In the past decade, literature regarding negative imaginary (NI) systems has flourished as well as its engineering applications (for some examples see [1] and the references therein). Since the positive feedback interconnection of an NI system with a strictly negative imaginary (SNI) is stable as long as certain conditions are satisfied [2], [3], [4], [5], [6], [7], [8], it is desired to synthesize a robust controller such that the closed-loop system becomes SNI (NI) when it is known that the uncertain system is NI (SNI respectively). Controller synthesis methods frequently require checking a property via a state-space characterization of the closedloop system and it is also highly desired to obtain an internally stable closed-loop system. However, controller synthesis methods do not guarantee a minimal state-space realization of the closed-loop system 'a priori'. For all these reasons, it is important to develop necessary and sufficient conditions based on non minimal state-space realizations of systems to determine whether a system is NI/SNI or not. These conditions are captured in results known as the NI lemma and the SNI lemma respectively. While the first versions of the SNI lemma [3], [9] considered minimal state-space realizations, [10] provided sufficient conditions, based on a non minimal state-space realization, to check if a system belongs to a subclass of SNI systems, known as strongly SNI (SSNI) systems. SSNI systems are SNI systems with particular properties at zero and infinity frequencies which restrict the SNI system class. Moreover, [11] offered sufficient conditions for a system to be SNI, but the matrix $A$ is required to be Hurwitz as an assumption instead of being

\footnotetext{
*This work was supported by the Engineering and Physical Sciences Research Council (EPSRC) [grant number EP/R008876/1]; and the Ecuadorian government's Secretaría de Educación Superior, Ciencia, Tecnología e Innovación (Senescyt). All research data supporting this publication are directly available within this publication.

${ }^{1}$ The authors are with the Control Systems Centre, School of Electrical and Electronic Engineering, University of Manchester, United Kingdom. Gabriela.Salcan@manchester.ac.uk, Alexander. Lanzon@manchester.ac.uk

${ }^{2}$ Gabriela Salcán is also with Escuela Superior Politécnica del Litoral, ESPOL, FIEC, Campus Gustavo Galindo Km 30.5 Vía Perimetral, P.O. Box 09-01-5863, Guayaquil, Ecuador. gapasalc@espol .edu.ec
}

implied by the conditions. On the other hand, [9] provides a NI lemma based on a minimal state-space realization, while [12] provides an NI Lemma without minimal assumptions based on linear matrix inequalities (LMIs). [12], [9] also offered a state-space feedback controller synthesis method based on algebraic Riccati equations (AREs) and LMIs respectively while [13] generalized results of [12] and offered an output feedback controller synthesis algorithm. But, the main drawback of all these controller synthesis methods ([12], [9], [13]) is that they only guarantee a system to be NI. In other words, the designed controllers allow the closedloop system to have poles on the $j \omega$ axis and hence they do not guarantee the internal stability of the closed-loop system. Furthermore, to the best of the author's knowledge, there are no contributions to render a closed-loop system SNI. Then, our motivation is to find conditions for the existence of controllers that render the closed-loop SNI and internally stable. Then, our main contributions are: 1) a set of necessary and sufficient conditions to check the SNI property for systems with non-minimal state-space realization, 2) a set of sufficient conditions for the existence of an output feedback controller such that the closed-loop system becomes SNI and internally stable and 3) a formula for such controller.

Notation: $\mathbb{R}, \mathbb{C}$ denotes the fields of Real and complex numbers respectively. $\mathbb{C}_{<0}$ represents the open left half plane of complex numbers. $\mathfrak{R}(s)$ stands for the real part of a complex number $s$ while $j$ represents the imaginary unit. $A^{n \times m}$ is a matrix with $n$ rows and $m$ columns. $I$ and 0 denotes the identity and zero matrices of appropriate dimensions respectively, however a subscript $n$ indicates a matrix of dimensions $n \times n . A^{T}, A^{*}, A^{\dagger}$ stand for the transpose, complex conjugate transpose and pseudo-inverse of $A$ respectively. $\mathscr{F}_{l}(G, K)$ denotes the lower linear fractional transformation of matrices $G$ and $K$. $\mathscr{R}$ denotes the set of all real, rational, proper transfer function matrices. Then, given $G \in \mathscr{R}, G=$ $\left[\begin{array}{l|l}A & B \\ \hline C & D\end{array}\right]$ is a shorthand of $G(s)=C(s I-A)^{-1} B+D$.

\section{PRELIMINARIES}

Here, we will give some background material that will help to streamline the understanding of the main results on this paper.

Definition 1: [14], [15] A real, rational transfer function matrix $G: \mathbb{C} \rightarrow \mathbb{C}^{m \times m}$ is negative imaginary (NI) if the following conditions are satisfied:

1) $G(s)$ has no poles in $\{s \in \mathbb{C}: \mathfrak{R}(s)>0\}$;

2) $j\left[G(j \omega)-G(j \omega)^{*}\right] \geq 0$ for all $\omega \in(0, \infty)$ except for the values of $\omega$ where $j \omega$ is a pole of $G(s)$; 
3) if $s=j \omega_{0}$ with $\omega_{0} \in(0, \infty)$ is a pole of $G(s)$, then it is a simple pole and the residue matrix of $j G(s)$, $K_{0}=\lim _{s \rightarrow j \omega_{0}}\left(s-j \omega_{0}\right) j G(s)$, is Hermitian and positive semidefinite;

4) if $s=0$ is a pole of $G(s)$, then it is at most a double pole and $\lim _{s \rightarrow 0} s^{2} G(s)$ is Hermitian and positive semidefinite;

5) if $s=\infty$ is a pole of $G(s)$, then it is at most a double pole and $\lim _{\omega \rightarrow \infty} \frac{G(j \omega)}{(j \omega)^{2}}$ is Hermitian and negative semidefinite.

Even though NI systems include non-rational systems [14], in this paper we will only consider NI transfer functions that are real and rational.

An important subset of the NI class are the strictly negative imaginary systems.

Definition 2: [2] A real, rational and proper transfer function $G: \mathbb{C} \rightarrow \mathbb{C}^{m \times m}$ is strictly negative imaginary (SNI) if

1) $G(s)$ has no poles in $\{s \in \mathbb{C}: \mathfrak{R}(s) \geq 0\}$;

2) $j\left[G(j \omega)-G(j \omega)^{*}\right]>0$ for all $\omega>0$.

The strict condition $j\left[G(j \omega)-G(j \omega)^{*}\right]>0$ is always violated at $\omega=0$ and $\omega=\infty$ since $G(0)=G(0)^{*}$ and $G(\infty)=$ $G(\infty)^{*}$ [2]. The authors in [15] claim to generalize the definition of SNI to include non-proper transfer functions. However, as we show in the following example, [15, Definition 3] which is supposed to characterize an SNI system does not fulfill the basic requirements of NI systems.

Example 1: Consider $G(s)=s^{3}$. It follows that $G(s)$ has no poles in $\{s \in \mathbb{C}: \Re(s) \geq 0\}$ and $j\left[G(j \omega)-G(j \omega)^{*}\right]=$ $2 \omega^{3}>0$ for all $\omega>0$. Then, according to [15, Definition $3]$, this non-proper transfer function matrix $G(s)$ is SNI. However, $G(s)$ violates condition 5) in Definition 1 since $s=\infty$ is a triple pole of $G(s)$. Thus $G(s)$ cannot be SNI.

NI systems are related to positive real (PR) systems which have been studied for a few decades and used in several applications. For more details in PR systems see [16], [17] and references therein.

Definition 3: The transfer matrix $F: \mathbb{C} \rightarrow \mathbb{C}^{m \times m}$ is positive real (PR) if:

1) $F(s)$ is analytic in $\{s \in \mathbb{C}: \mathfrak{R}(s)>0\}$;

2) $F(s)$ is real for all positive real $s$;

3) $F(s)+F(s)^{*} \geq 0$ for all $\{s \in \mathbb{C}: \Re(s)>0\}$.

We will use the following standard definition of SPR from [17].

Definition 4: The transfer function $F: \mathbb{C} \rightarrow \mathbb{C}^{m \times m}$ is strictly positive real (SPR) if for some $\varepsilon>0$, the transfer function $F(s-\varepsilon)$ is PR.

The following lemma shows the duality in NI systems. In other words, to test stability or synthesize a controller, one can obtain the results for $G$ from its dual object $G^{T}$ if they are available, and vice versa.

Lemma 1: Let $G: \mathbb{C} \rightarrow \mathbb{C}^{m \times m}$ be a real, rational, proper transfer function. Then $G(s)$ is NI (respectively SNI) if and only if $G(s)^{T}$ is NI (respectively SNI).

Proof: This trivially follows from Definition 1 (respectively Definition 2 ) and the fact that $A \geq($ respectively $>) 0$ if and only if $A^{T} \geq($ respectively $>) 0$.

\section{NI AND SNI LEMMAS WITH NON-MINIMAL STATE-SPACE REALIZATIONS}

\section{A. NI lemma with non-minimal state-space realization}

In this subsection we present a set of sufficient conditions to check the NI property of systems. The main difference between our results and those from [9], [18] is that we do not require a minimal state-space realization. Furthermore, we relaxed the non-singularity assumption $\operatorname{det}(A) \neq 0$ in [12], which underpins the NI synthesis problem in [12], [13].

Lemma 2: Let $\left[\begin{array}{c|c}A & B \\ \hline C & D\end{array}\right]$ be a state-space realization of a real, rational transfer function $G: \mathbb{C} \rightarrow \mathbb{C}^{m \times m}$ with $R=C B+$ $(C B)^{*}>0$. Then, the following statements are equivalent:

1) There exists a real $X=X^{*}$ such that $A^{*} X+X A+(C A-$ $\left.B^{*} X\right)^{*} R^{-1}\left(C A-B^{*} X\right)=0$.

2) There exist real matrices $X=X^{*}, L$ and a nonsingular $W$ such that

$$
\begin{aligned}
A^{*} X+X A & =-L^{*} L, \\
C A-B^{*} X & =W^{*} L, \\
C B+B^{*} C^{*} & =W^{*} W .
\end{aligned}
$$

3) When $A$ is nonsingular, there exist real matrices $X=X^{*}$ and $L$ such that $A^{*} X+X A=-L^{*} L$ and $C=-B^{*} A^{-*} X$. Moreover, if any of these statements hold with $X=X^{*} \geq 0$ and $D=D^{*}$, then $G(s)$ is NI.

Proof: 1) $\Longrightarrow 2$ ) Suppose $A \in \mathbb{R}^{n \times n}$. Note that $R$ loses rank when $n<m$, therefore $n \geq m$. Then, there exist $L_{1} \in$ $\mathbb{R}^{k \times n}$ and a nonsingular $W \in \mathbb{R}^{m \times m}$ such that $A^{*} X+X A=$ $-L_{1}^{*} L_{1}$ and $C B+B^{*} C^{*}=W^{*} W$, where $k=\operatorname{rank}\left(A^{*} X+X A\right) \leq$ $m$. So we have $L_{1}^{*} L_{1}=\left(C A-B^{*} X\right)^{*} W^{-1} W^{-*}\left(C A-B^{*} X\right)$. As a consequence, there exists a $U \in \mathbb{R}^{m \times k}$ such that $U^{*} U=I$ and $W^{-*}\left(C A-B^{*} X\right)=U L_{1}$. Next, by letting $L=U L_{1}$ we get (1), (2) and (3).

2) $\Longrightarrow 1$ ) Follows by simple replacement.

2) $\Longleftrightarrow 3$ ) Equations (1), (2) and (3) can be rewritten as

$$
0 \geq-\left[\begin{array}{c}
L^{*} \\
W^{*}
\end{array}\right]\left[\begin{array}{ll}
L & W
\end{array}\right]=\left[\begin{array}{c}
A^{*} \\
B^{*}
\end{array}\right]\left[\begin{array}{ll}
X & -C^{*}
\end{array}\right]+\left[\begin{array}{c}
X \\
-C
\end{array}\right]\left[\begin{array}{ll}
A & B
\end{array}\right],
$$

which, when $A$ is nonsingular, is equivalent to

$$
\begin{aligned}
0 \geq & {\left[\begin{array}{cc}
I & 0 \\
B^{*} A^{-*} & -I
\end{array}\right]\left\{\left[\begin{array}{l}
A^{*} \\
B^{*}
\end{array}\right]\left[\begin{array}{ll}
X & -C^{*}
\end{array}\right]\right.} \\
& \left.+\left[\begin{array}{c}
X \\
-C
\end{array}\right]\left[\begin{array}{ll}
A & B
\end{array}\right]\right\}\left[\begin{array}{cc}
I & A^{-1} B \\
0 & -I
\end{array}\right] \\
= & {\left[\begin{array}{cc}
A^{*} X+X A & A^{*}\left(C^{*}+X A^{-1} B\right) \\
\left(C+B^{*} A^{-*} X\right) A & 0
\end{array}\right] . }
\end{aligned}
$$

Therefore, $C=-B^{*} A^{-*} X$. Finally, note that (1), (2) and (3) with $X \geq 0$ implies that $\left[\begin{array}{c|c}A & B \\ \hline C A & C B\end{array}\right]=s(G(s)-D)$ is PR via [19, Corollary 3] or, equivalently when $D=D^{*}, G(s)$ is NI according to [20], [15]. Here concludes the proof.

In contrast to statement 3) in Lemma 2, the conditions in the next lemma reduce to previous conditions in the literature. Furthermore, $A$ can be either singular or nonsingular. 
Lemma 3: Let $\left[\begin{array}{c|c}A & B \\ \hline C & D\end{array}\right]$ be a state-space realization of a real, rational transfer function $G: \mathbb{C} \rightarrow \mathbb{C}^{m \times m}$ with $R=C B+$ $(C B)^{*}>0$. Then, the following statements are equivalent:

1) There exists a real $Y=Y^{*}$ such that $A Y+Y A^{*}+(B-$ $\left.Y A^{*} C^{*}\right) R^{-1}\left(B-Y A^{*} C^{*}\right)^{*}=0$.

2) There exist real matrices $Y=Y^{*}, Q$ and a nonsingular $W$ such that

$$
\begin{aligned}
A Y+Y A^{*} & =-Q^{*} Q, \\
B^{*}-C A Y & =W^{*} Q, \\
C B+B^{*} C^{*} & =W^{*} W .
\end{aligned}
$$

3) There exist real matrices $Y=Y^{*}$ and $Q$ such that $A Y+$ $Y A^{*}=-Q^{*} Q$ and $B=-A Y C^{*}$.

Moreover, if any of these statements hold with $Y=Y^{*} \geq 0$ and $D=D^{*}$, then $G(s)$ is NI.

Proof: By following closely the proof of Lemma 2 with appropriate modifications in pertinent statements, we easily obtain the equivalence between 1) and 2). The equivalence between 2) and 3) follows on noting that

$$
\begin{aligned}
0 \geq-\left[\begin{array}{c}
Q^{*} \\
W^{*}
\end{array}\right] & {\left[\begin{array}{c}
Q^{*} \\
W^{*}
\end{array}\right]^{*}=\left[\begin{array}{l}
I \\
C
\end{array}\right]\left[\begin{array}{ll}
A Y & -B
\end{array}\right]+\left[\begin{array}{c}
Y A^{*} \\
-B^{*}
\end{array}\right]\left[\begin{array}{ll}
I & C^{*}
\end{array}\right] } \\
\Longleftrightarrow 0 & \geq\left[\begin{array}{cc}
I & 0 \\
-C & I
\end{array}\right]\left\{\left[\begin{array}{l}
I \\
C
\end{array}\right]\left[\begin{array}{cc}
A Y & -B
\end{array}\right]\right. \\
& \left.+\left[\begin{array}{c}
Y A^{*} \\
-B^{*}
\end{array}\right]\left[\begin{array}{ll}
I & C^{*}
\end{array}\right]\right\}\left[\begin{array}{cc}
I & -C^{*} \\
0 & I
\end{array}\right] \\
& =\left[\begin{array}{cc}
A Y+Y A^{*} & -\left(B+A Y C^{*}\right) \\
-\left(B+A Y C^{*}\right)^{*} & 0
\end{array}\right] .
\end{aligned}
$$

Similarly to the proof of Lemma 2 it follows by (4), (5), (6) and $Y=Y \geq 0$ that $\left[\begin{array}{l|l}A^{*} & A^{*} C^{*} \\ \hline B^{*} & B^{*} C^{*}\end{array}\right]=(s(G(s)-D))^{T}$ is PR, or equivalently, $G(s)^{T}$ is NI, which in turn is equivalent to $G(s)$ is NI by Lemma 1 .

Remark 1: When $A$ is nonsingular, Lemma 3 is obtained trivially from Lemma 2 via the following substitutions $A \rightarrow$ $A^{*}, B \rightarrow A^{*} C^{*}, C \rightarrow B^{*} A^{-*}, X \rightarrow Y, L \rightarrow Q$ and simple algebraic rearrangements.

\section{B. SNI Lemma with non-minimal state-space realization}

In this subsection we show the extra conditions that a system should satisfy in order to guarantee the strictness of the NI class, based on the NI lemmas shown in the previous subsection. Furthermore, we offer conditions that are not only sufficient, but also necessary. Note that the state-space realization in the following theorem does not need to be minimal, thus generalizing results of [9].

Theorem 1 (SNI Lemma): Let $\left[\begin{array}{l|l}A & B \\ \hline C & D\end{array}\right]$ be a state-space realization of the real, rational transfer function $G: \mathbb{C} \rightarrow$ $\mathbb{C}^{m \times m}$, with $R=C B+(C B)^{*}>0$. Then the following statements are equivalent:

1) $G(s)$ is SNI and $A$ is Hurwitz.

2) $D=D^{*}, A$ is Hurwitz and the Hamiltonian matrix

$$
H=\left[\begin{array}{cc}
A-B R^{-1} C A & B R^{-1} B^{*} \\
-A^{*} C^{*} R^{-1} C A & -\left(A-B R^{-1} C A\right)^{*}
\end{array}\right]
$$

has no eigenvalues on the $j \omega$-axis except at the origin.

3) $D=D^{*}, \operatorname{det}(A) \neq 0$ and there exists a real $X=X^{*} \geq 0$ such that

$$
A^{*} X+X A+\left(C A-B^{*} X\right)^{*} R^{-1}\left(C A-B^{*} X\right)=0
$$

and $\sigma\left(A-B R^{-1}\left(C A-B^{*} X\right)\right) \subset \mathbb{C}_{<0} \cup\{0\}$.

4) $D=D^{*}$, A is Hurwitz and there exist a real $X=X^{*}$ and a real $L$ such that

$$
\begin{aligned}
A^{*} X+X A & =-L^{*} L, \\
C & =-B^{*} A^{-*} X
\end{aligned}
$$

and $\operatorname{rank}(M(j \omega))=m$ for all $\omega \in(0, \infty)$, where $M(s)=$ $L A^{-1}(s I-A)^{-1} B$.

5) $D=D^{*}, \operatorname{det}(A) \neq 0$ and there exists a real $Y=Y^{*} \geq 0$ such that

$$
A Y+Y A^{*}+\left(B-Y A^{*} C^{*}\right) R^{-1}\left(B-Y A^{*} C^{*}\right)^{*}=0
$$

and $\sigma\left(A-\left(B-Y A^{*} C^{*}\right) R^{-1} C A\right) \subset \mathbb{C}_{<0} \cup\{0\}$.

6) $D=D^{*}, \mathrm{~A}$ is Hurwitz and there exist a real $Y=Y^{*}$ and a real $Q$ such that

$$
\begin{aligned}
A Y+Y A^{*} & =-Q^{*} Q, \\
B & =-A Y C^{*}
\end{aligned}
$$

and $\operatorname{rank}(N(j \omega))=m$ for all $\omega \in(0, \infty)$, where $N(s)=$ $C(s I-A)^{-1} Q^{*}$.

Proof: The proof will be published elsewhere

Remark 2: When any of these conditions hold, the almost stabilizing solutions $X=X^{*} \geq 0$ and $Y=Y^{*} \geq 0$ to the AREs (8) and (11) respectively are unique. Furthermore, the partial multiplicities (i.e. the sizes of the Jordan blocks associated to which an eigenvalue belongs) of the zero eigenvalues of the Hamiltonian (7) are all even [21], [22].

Remark 3: Note that $C(I-Y X)=0$ and $(I-Y X) A^{-1} B=$ 0 from statements 4 ) and 6).

C. A strongly SNI Lemma with non-minimal state-space realization

The class of SNI systems such that $\lim _{\omega \rightarrow 0^{+}} \frac{1}{\omega} j\left[G(j \omega)-G(j \omega)^{*}\right]>0$, which in the scalar case means that the phase of $G(j \omega)$ strictly decreases as frequency increases form $\omega=0$ [10], and $\lim _{\omega \rightarrow \infty} j\left[G(j \omega)-G(j \omega)^{*}\right]>0$, has interesting spectral properties and are known as strongly strictly negative imaginary (SSNI) systems [10]. As shown in the next theorem, we can transform the ARE associated with an SSNI system to an ARE with an almost stabilizing solution. Thus, we offer an ARE with better numerical properties and hence we are avoiding the challenging numerical problems present in the computation of solutions of AREs with singular Hamiltonians [21], such as the Hamiltonians associated to SNI systems.

Theorem 2 (Strongly SNI Lemma): Let $\left[\begin{array}{l|l}A & B \\ \hline C & D\end{array}\right]$ be a state-space realization of the real transfer function $G: \mathbb{C} \rightarrow$ $\mathbb{C}^{m \times m}$ with $R=C B+(C B)^{*}>0$. Then, the following statements are equivalent: 
1) $G(s)$ is SNI with $A$ Hurwitz and $\lim _{\omega \rightarrow 0^{+}} \frac{1}{\omega} j[G(j \omega)-$ $\left.G(j \omega)^{*}\right]>0$.

2) $D=D^{*}, \operatorname{det}(A) \neq 0$ and there exists a real $X=X^{*} \geq 0$ such that (8) holds and the matrix $A-B R^{-1}\left(C A-B^{*} X\right)$ has all its eigenvalues in $\mathbb{C}_{<0}$ except $m$ eigenvalues at the origin.

3) $D=D^{*}, \operatorname{det}(A) \neq 0$ and there exists a real $X=X^{*} \geq 0$ such that $C=-B^{*} A^{-*} X$,

$$
\widetilde{A}^{*} X+X \widetilde{A}+X \widetilde{B} X+\widetilde{C}=0,
$$

and $\sigma(\widetilde{A}+\widetilde{B} X) \subset \mathbb{C}_{<0}$, where for any $\eta<0$,

$$
\begin{aligned}
& \widetilde{A}=A-B R^{-1} C A+\eta A^{-1} B\left(B^{\dagger} A-C\right), \\
& \widetilde{B}=B R^{-1} B^{*}-\eta A^{-1} B B^{*} A^{-*}, \\
& \widetilde{C}=A^{*} C^{*} R^{-1} C A-\eta\left[C^{*} C-C^{*} B^{\dagger} A-\left(B^{\dagger} A\right)^{*} C\right] .
\end{aligned}
$$

4) $D=D^{*}, \operatorname{det}(A) \neq 0$ and there exists a real $Y=$ $Y^{*} \geq 0$ such that (11) holds and the matrix $A-$ $\left(B-Y A^{*} C^{*}\right) R^{-1} C A$ has all its eigenvalues in $\mathbb{C}_{<0}$ except $m$ eigenvalues at the origin.

5) $D=D^{*}, \operatorname{det}(A) \neq 0$ and there exists a real $Y=Y^{*} \geq 0$ such that $B=-A Y C^{*}$,

$$
\Delta Y+Y \Delta^{*}+Y \Theta Y+\Gamma=0,
$$

and $\sigma(\Delta+Y \Theta) \subset \mathbb{C}_{<0}$, where for any $\eta<0$,

$$
\begin{aligned}
& \Delta=A-B R^{-1} C A+\eta\left(C^{\dagger}-A^{-1} B\right) C, \\
& \Theta=A^{*} C^{*} R^{-1} C A-\eta C^{*} C, \\
& \Gamma=B R^{-1} B^{*}-\eta A^{-1}\left[B B^{*}-B C^{\dagger *} A^{*}-A C^{\dagger} B^{*}\right] A^{-*} .
\end{aligned}
$$

Furthermore, the matrix $X$ in conditions 2) and 3) is the same; so is the matrix $Y$ in conditions 4) and 5).

Proof: The proof will be published elsewhere

\section{Illustrative example}

The following example illustrates the usefulness of the SNI lemmas derived in this section. Consider the transfer function matrix

$$
G(s)=\left[\begin{array}{ll}
\frac{2\left(7 s^{2}+10 s+4\right)}{(s+1)^{2}(s+2)} & \frac{-\left(3 s^{2}+12 s+4\right)}{(s+1)^{2}(s+2)} \\
\frac{-4(2 s+1)}{(s+1)^{2}(s+2)} & \frac{2\left(s^{2}+6 s+10\right)}{(s+1)^{2}(s+2)}
\end{array}\right]
$$

with a state-space realization

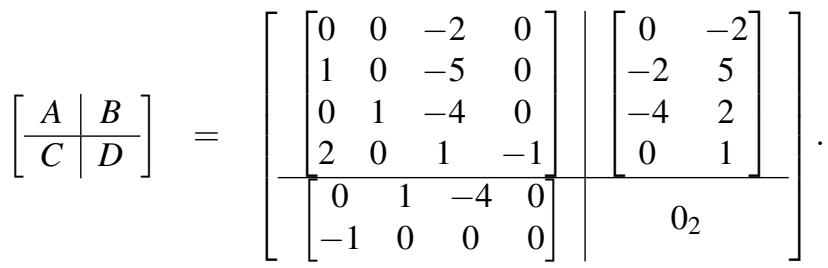

Observe that $C B+B^{*} C^{*}=\left[\begin{array}{cc}28 & -3 \\ -3 & 4\end{array}\right]>0$ and this realization is non-minimal since the pair $(C, A)$ has an unobservable mode $(s=-1)$. It is easy to check that $A$ is Hurwitz and $\sigma(H)=\sigma\left(\left[\begin{array}{cc}A-B R^{-1} C A & B R^{-1} B^{*} \\ -A^{*} C^{*} R^{-1} C A & -\left(A-B R^{-1} C A\right)^{*}\end{array}\right]\right)=$
$\{-1,0,0,0,0,0,0,1\}$, therefore $G(s)$ is SNI by Theorem 1 . In fact,

$$
X=\left[\begin{array}{cccc}
0.1250 & -0.0625 & 0.1250 & 0 \\
-0.0625 & 0.2813 & -1.0625 & 0 \\
0.1250 & -1.0625 & 5.1250 & 0 \\
0 & 0 & 0 & 0
\end{array}\right] \geq 0
$$

is the unique solution to (8) with $\sigma\left(A-B R^{-1}(C A-\right.$ $\left.\left.B^{*} X\right)\right)=\{-1,0,0,0\}$. Then, by Theorem 2 $\lim _{\omega \rightarrow 0^{+}} \frac{1}{\omega} j\left[G(j \omega)-G(j \omega)^{*}\right]$ is not a positive definite matrix, since $A-B R^{-1}\left(C A-B^{*} X\right)$ has more than $m=2$ zero eigenvalues at the origin. This is confirmed since $\lim _{\omega \rightarrow 0^{+}} \frac{1}{\omega} j\left[G(j \omega)-G(j \omega)^{*}\right]=\left[\begin{array}{cc}0 & 0 \\ 0 & 38\end{array}\right] \geq 0$. It is also simple to verify that this $X$ satisfies (9) and (10) and gives $L=\left[\begin{array}{cccc}0.0903 & -0.1079 & 0.7939 & -0.0647 \\ -0.1079 & 0.5374 & -1.2244 & -0.1168 \\ 0.7939 & -1.2244 & 7.1638 & -0.4372 \\ -0.0647 & -0.1168 & -0.4372 & 0.1384\end{array}\right]$ from (9), which in turn gives $M(s)$ with no transmission zeros. Moreover, $Y=\left[\begin{array}{cccc}10 & 6 & 1 & 20 \\ 6 & 20 & 4 & 32 \\ 1 & 4 & 1 & 7 \\ 20 & 32 & 7 & 76.8932\end{array}\right]>0$ is the unique solution to (11) with $\sigma\left(A-\left(B-Y A^{*} C^{*}\right) R^{-1} C A\right)=$ $\{-1,0,0,0\}$. Again, it is simple to verify that this $Y$ satisfies (13) and gives $Q=\left[\begin{array}{cccc}0.7980 & -0.3668 & 0 & 1.7968 \\ -0.3668 & 4.5046 & 0 & 2.7522 \\ 0 & 0 & 0 & 0 \\ 1.7968 & 2.7522 & 0 & 6.9988\end{array}\right]$ from (12), which in turn gives $N(s)$ with no transmission zeros. Finally, $C(I-Y X)=0$ and $(I-Y X) A^{-1} B=0$.

Remark 4: Observe that $X=Y^{-1}$ also solves (8), but it is not an almost stabilizing solution to ARE (8) since $\sigma\left(A-B R^{-1}\left(C A-B^{*} Y^{-1}\right)\right)=\{1,0,0,0\}$. This is consistent with Remark 2 in regard to the uniqueness of the almost stabilizing solution of (8).

\section{SNI CONTROL PROBLEM}

Consider the uncertain linear system depicted in Fig. 1 where the uncertainty $\Delta(s)$ is NI and the generalized plant $G$ is described by

$$
\begin{aligned}
& \dot{x}=A x+B_{1} w+B_{2} u \\
& z=C_{1} x+D_{11} w+D_{12} u \\
& y=C_{2} x+D_{21} w+D_{22 u}
\end{aligned}
$$

where $x$ is the state of the plant, $w$ is the disturbance acting on the system, $u$ is the control input, $z$ is the controlled output signal, $y$ is the measurement output, $A \in \mathbb{R}^{n \times n}, B_{1} \in \mathbb{R}^{n \times m}$ $B_{2} \in \mathbb{R}^{n \times q}, C_{1} \in \mathbb{R}^{m \times n}, C_{2} \in \mathbb{R}^{p \times n}, D_{11} \in \mathbb{R}^{m \times m}, D_{12} \in \mathbb{R}^{m \times q}$, $D_{21} \in \mathbb{R}^{p \times m}, D_{22} \in \mathbb{R}^{p \times q}$ and $q \leq m, p \leq m$.

Our aim is to find conditions for the existence of a controller $K(s)$ such that the closed-loop system $\mathscr{F}_{l}(G, K)$ is SNI and internally stable and provide a formula for such controller. 


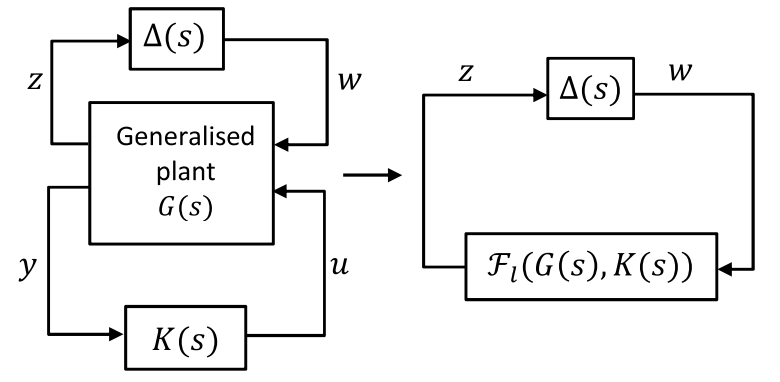

Fig. 1. Feedback control of an uncertain system

\section{A. Static state-feedback}

If all the system states are available to the controller, then we can design a state-feedback controller such that the closed-loop system is SNI as shown in the following result.

Note that in this case, the generalized plant $G$ becomes

$$
\begin{aligned}
\dot{x} & =A x+B_{1} w+B_{2} u \\
z & =C_{1} x+D_{11} w \\
y & =x
\end{aligned}
$$

and the static state-feedback control law is given by $u=K x$.

Theorem 3: Consider the generalized plant $G$ in (16) where $C_{1} B_{2}$ is full column rank, and $\left(A, B_{2}\right)$ is stabilizable. Let $R=C_{1} B_{1}+B_{1}^{*} C_{1}^{*}>0$ and suppose that

1) $D_{11}=D_{11}^{*}$;

2) there exists a real $X \geq 0$ such that

$$
A_{x}^{*} X+X A_{x}+X R_{x} X+Q_{x}=0,
$$

where

$$
\begin{aligned}
A_{x}= & \left(I-B_{1} R^{-1} C_{1}\right)\left[I-B_{2}\left(B_{2}^{*} C_{1}^{*} R^{-1} C_{1} B_{2}\right)^{-1}\right. \\
& \left.B_{2}^{*} C_{1}^{*} R^{-1} C_{1}\right] A, \\
R_{x}= & B_{1} R^{-1} B_{1}^{*}-\left(I-B_{1} R^{-1} C_{1}\right) B_{2}\left(B_{2}^{*} C_{1}^{*} R^{-1}\right. \\
& \left.C_{1} B_{2}\right)^{-1} B_{2}^{*}\left(I-B_{1} R^{-1} C_{1}\right)^{*}, \\
Q_{x}= & A^{*} C_{1}^{*} R^{-1}\left[R-C_{1} B_{2}\left(B_{2}^{*} C_{1}^{*} R^{-1} C_{1} B_{2}\right)^{-1} B_{2}^{*}\right. \\
& \left.C_{1}^{*}\right] R^{-1} C_{1} A .
\end{aligned}
$$

Then, the state-feedback controller $u=F x$ generates an NI closed-loop system

$$
\left[\begin{array}{c|c}
A+B_{2} F & B_{1} \\
\hline C_{1} & D_{11}
\end{array}\right]
$$

where

$$
F=-\left(B_{2}^{*} C_{1}^{*} R^{-1} C_{1} B_{2}\right)^{-1} B_{2}^{*}\left[X+C_{1}^{*} R^{-1}\left(C_{1} A-B_{1}^{*} X\right)\right] .
$$

Moreover, if in addition $\sigma\left(A_{x}+R_{x} X\right) \subset \mathbb{C}_{<0} \cup\{0\}$ and $\operatorname{det}\left(A+B_{2} F\right) \neq 0$, then the closed-loop system (21) is SNI with $A+B_{2} F$ Hurwitz.

Proof: The proof will be published elsewhere

Remark 5: Note that when $A$ is nonsingular the columns of $A^{-1} B_{2}$ belongs to $\operatorname{ker} A_{x} \cap \operatorname{ker} Q_{x}$. On the other hand, when $A$ is singular there exists $0 \neq v \in \mathbb{R}^{n}$ such that $A v=0$, thus $v \subset \operatorname{ker} A_{x} \cap \operatorname{ker} Q_{x}$. Consequently, $\operatorname{ker} A_{x} \cap \operatorname{ker} Q_{x}$ is not an empty set. Then, it follows that the ARE (17) has a singular Hamiltonian and its solution may not be unique. However, we can find some of these solutions by following the procedure in [23]. Singular Hamiltonians arise in NI controller synthesis.

Remark 6: The assumption that $\left(A, B_{2}\right)$ is stabilizable is not needed to design a feedback gain $K$ such that $\mathscr{F}_{l}(G, K)$ is NI. This assumption is however required for $\mathscr{F}_{l}(G, K)$ to be internally stable.

\section{B. Dynamic output feedback}

Consider the generalized plant $G$ in (15). The controller $K: \mathbb{C} \rightarrow \mathbb{C}^{q \times p}$ is described by

$$
\begin{aligned}
\dot{x}_{k} & =A_{k} x_{k}+B_{k} y \\
u & =C_{k} x_{k}+D_{k} y
\end{aligned}
$$

where $x_{k}$ is the state of the controller, $A_{k} \in \mathbb{R}^{k \times k}, B_{k} \in \mathbb{R}^{k \times p}$, $C_{k} \in \mathbb{R}^{q \times k}$ and $D_{k} \in \mathbb{R}^{q \times p}$.

Suppose the generalized plant state-space matrices in (15) satisfy the following assumptions:

(A1) $\left(A, B_{2}\right)$ is stabilizable and $\left(C_{2}, A\right)$ is detectable;

(A2) $C_{1} B_{2}$ is full column rank, $D_{21}$ has full row rank;

(A3) $D_{12}=0$ and $D_{22}=0$;

(A4) $C_{1} B_{1}+B_{1}^{*} C_{1}^{*}>0$.

Assumption (A1) is necessary for internal stability. Assumption (A2) is analogous to the corresponding assumption in LQG/ $\mathscr{H}_{\infty} / \mathrm{SPR}$ synthesis [24], [25] for the system $s\left(G(s)-D_{11}\right)=\left[\begin{array}{c|cc}A & B_{1} & B_{2} \\ \hline C_{1} & C_{1} B_{1} & C_{1} B_{2} \\ C_{2} & D_{21} & D_{22}\end{array}\right]$. The assumption that $C_{1} B_{2}$ is full column rank in (A2) means that the transfer function from $u$ to $z$ has relative degree of one. The assumptions in (A3) can easily be relaxed as shown in [26, Lemma 5]. Assumption (A4) is necessary for the synthesis method presented in the following result.

Theorem 4: Consider the generalized plant $G$ in (15) and let the assumptions (A1) to (A4) hold. Let $R=C_{1} B_{1}+B_{1}^{*} C_{1}^{*}$ and suppose that

1) $D_{11}=D_{11}^{*}$;

2) there exists a real $X \geq 0$ such that $A_{x}^{*} X+X A_{x}+X R_{x} X+$ $Q_{x}=0$, where $A_{x}, R_{x}$ and $Q_{x}$ are defined in (18), (19) and (20) respectively;

3) there exists a real $Y \geq 0$ such that $A_{y} Y+Y A_{y}^{*}+Y R_{y} Y+$ $Q_{y}=0$ and $\sigma\left(A_{y}+Y R_{y}\right) \subset \mathbb{C}_{<0}$, where

$$
\begin{aligned}
A_{y}= & \left(I-B_{1} R^{-1} C_{1}\right) A-B_{1} R^{-1} D_{21}^{*}\left(D_{21} R^{-1} D_{21}^{*}\right)^{-1} \\
& \left(C_{2}-D_{21} R^{-1} C_{1} A\right) \\
R_{y}= & A^{*} C_{1}^{*} R^{-1} C_{1} A-\left(C_{2}-D_{21} R^{-1} C_{1} A\right)^{*} \\
& \left(D_{21} R^{-1} D_{21}^{*}\right)^{-1}\left(C_{2}-D_{21} R^{-1} C_{1} A\right), \\
Q_{y}= & B_{1} R^{-1}\left[R-D_{21}^{*}\left(D_{21} R^{-1} D_{21}^{*}\right)^{-1} D_{21}\right] R^{-1} B_{1}^{*} ;
\end{aligned}
$$

4) $\rho(Y X)<1$. 
Then, the closed-loop system $\mathscr{F}_{l}(G, K)$ is NI when the controller

$$
K=\left[\begin{array}{c|c}
A+B_{2} F+(I-Y X)^{-1} L C_{2}+\Psi & -(I-Y X)^{-1} L \\
\hline F & 0
\end{array},\right.
$$

where $F$ is defined in (22) and

$$
\begin{aligned}
L & =-\left[Y C_{2}^{*}+\left(B_{1}^{*}-C_{1} A Y\right)^{*} R^{-1} D_{21}^{*}\right]\left(D_{21} R^{-1} D_{21}^{*}\right)^{-1} ; \\
\Psi & =-\left[B_{1}+(I-Y X)^{-1} L D_{21}\right] R^{-1}\left[C_{1}\left(A+B_{2} F\right)-B_{1}^{*} X\right] .
\end{aligned}
$$

If, in addition, $\sigma\left(A_{x}+R_{x} X\right) \subset \mathbb{C}_{<0} \cup\{0\} \quad$ and $\operatorname{det}\left(\left[\begin{array}{cc}A+B_{2} F & -B_{2} F \\ -\Psi & A+\Psi-B_{k} C_{2}\end{array}\right]\right) \neq 0$, then $\mathscr{F}_{l}(G, K)$ is SNI and the associated linear fractional interconnection is internally stable.

Proof: The proof will be published elsewhere

Remark 7: The procedure to synthesize a controller for the SNI problem is as follows: 1) verify that $D_{11}$ is symmetric, 2) find the unique solution of the ARE in the third condition of Theorem 4, 3) find all the solutions to the ARE in the second condition of Theorem 4, 4) from all the solutions found in step 3 check which of them satisfy $\sigma\left(A_{x}+R_{x} X\right) \subset \mathbb{C}_{<0} \cup\{0\}, \operatorname{det}\left(A_{c}\right) \neq 0$ and the fourth condition of Theorem 4 . It is worth mentioning that there may be more than one controller which solves this problem as pointed out in Remark 5.

\section{CONCLuSions}

NI and SNI lemmas for systems with non minimal statespace representation were derived in this work. These results are useful not only for controller synthesis but also for robust stability analysis. Then, we derived some sufficient conditions for the existence of a controller such that the closed-loop is SNI and also internally stable. Although these conditions do not seem to be conservative, necessary conditions for the SNI controller synthesis remain an open problem.

\section{REFERENCES}

[1] I. R. Petersen, "Negative imaginary systems theory and applications," Annual Reviews in Control, vol. 42, pp. 309-318, 2016.

[2] A. Lanzon and I. R. Petersen, "Stability robustness of a feedback interconnection of systems with negative imaginary frequency response," IEEE Transactions on Automatic Control, vol. 53, no. 4, pp. 10421046, May 2008.

[3] J. Xiong, I. R. Petersen, and A. Lanzon, "A negative imaginary lemma and the stability of interconnections of linear negative imaginary systems," IEEE Transactions on Automatic Control, vol. 55, no. 10, pp. 2342-2347, Oct 2010.

[4] S. Patra and A. Lanzon, "Stability analysis of interconnected systems with "mixed" negative-imaginary and small-gain properties," IEEE Transactions on Automatic Control, vol. 56, no. 6, pp. 1395-1400, Jun 2011.

[5] M. A. Mabrok, A. G. Kallapur, I. R. Petersen, and A. Lanzon, "Generalizing negative imaginary systems theory to include free body dynamics: Control of highly resonant structures with free body motion," IEEE Transactions on Automatic Control, vol. 59, no. 10, pp. 2692-2707, Oct 2014.

[6] P. Bhowmick and S. Patra, "On input-output negative-imaginary systems and an output strict negative-imaginary lemma," in Proceedings of the 2nd IEEE Indian Control Conference, Hyderabad, India, 2016, pp. 176-181.

[7] A. Lanzon and H. J. Chen, "Feedback stability of negative imaginary systems," IEEE Transactions on Automatic Control, vol. 62, no. 11, pp. 5620-5633, Nov 2017.
[8] A. Ferrante, A. Lanzon, and L. Ntogramatzidis, "Discrete-time negative imaginary systems," Automatica, vol. 79, pp. 1-10, May 2017.

[9] M. Mabrok, A. G. Kallapur, I. R. Petersen, and A. Lanzon, "A generalized negative imaginary lemma and Riccati-based static statefeedback negative imaginary synthesis," Systems \& Control Letters, vol. 77, pp. 63-68, Mar 2015.

[10] A. Lanzon, Z. Song, S. Patra, and I. R. Petersen, "A strongly strict negative-imaginary lemma for non-minimal linear systems," Соттиnications in Information and Systems, vol. 11, no. 2, pp. 139-152, 2011.

[11] J. Xiong, A. Lanzon, and I. R. Petersen, "Descriptor systems statespace conditions to guarantee negative imaginary properties without minimality restrictions," in Proceedings of the 54th IEEE Conference on Decision and Control, Osaka, Japan, 2015, pp. 2707-2712.

[12] Z. Song, A. Lanzon, S. Patra, and I. R. Petersen, "A negativeimaginary lemma without minimality assumptions and robust statefeedback synthesis for uncertain negative-imaginary systems," Systems \& Control Letters, vol. 61, pp. 1269-1276, Dec 2012.

[13] J. Xiong, J. Lam, and I. R. Petersen, "Output feedback negative imaginary synthesis under structural constraints," Automatica, vol. 71, pp. 222-228, Sep 2016.

[14] A. Ferrante, A. Lanzon, and L. Ntogramatzidis, "Foundations of not necessarily rational negative imaginary systems theory: Relations between classes of negative imaginary and positive real systems," IEEE Transactions on Automatic Control, vol. 61, no. 10, pp. 3052-3057, Oct 2016.

[15] M. Liu and J. Xiong, "On non-proper negative imaginary systems," Systems and Control Letters, vol. 88, pp. 47-53, Feb 2016.

[16] B. D. O. Anderson and S. Vongpanitlerd, Network Analysis and Synthesis: A Modern Systems Theory Approach. Englewood Cliffs, NJ, USA: Prentice Hall, 1973.

[17] B. Brogliato, R. Lozano, B. Maschke, and O. Egeland, Dissipative systems analysis and control: Theory and Applications, 2 nd ed. London, UK: Springer-Verlag London, 2007.

[18] M. A. Mabrok, A. G. Kallapur, I. R. Petersen, and A. Lanzon, "Spectral conditions for negative imaginary systems with applications to nanopositioning," IEEE/ASME Transactions on Mechatronics, vol. 19, no. 3, pp. 895-903, Jun 2014.

[19] R. Scherer and W. Wendler, "A generalization of the positive real lemma," IEEE Transactions on Automatic Control, vol. 39, no. 4, pp. 882-886, Apr 1994.

[20] A. Ferrante and L. Ntogramatzidis, "Some new results in the theory of negative imaginary systems with symmetric transfer matrix function," Automatica, vol. 49, no. 7, pp. 2138-2144, Jul 2013.

[21] D. A. Bini, B. Iannazzo, and B. Meini, Numerical Solution of Algebraic Riccati Equations. Philadelphia, PA, USA: Society for Industrial and Applied Mathematics, 2012.

[22] P. Lancaster and L. Rodman, Algebraic Riccati Equations. Oxford, UK: Clarendon Press, 1995.

[23] V. Larin, "Algorithm for solving algebraic Riccati equation which has singular Hamiltonian matrix," Systems \& Control Letters, vol. 36, pp. 231-239, 1999.

[24] J. Doyle, K. Glover, P. Khargonekar, and B. Francis, "State-space solutions to standard $\mathscr{H}_{2}$ and $\mathscr{H}_{\infty}$ control problems," IEEE Transactions on Automatic Control, vol. 34, no. 8, pp. 831-847, 1989.

[25] W. Sun, P. P. Khargonekar, and D. Shim, "Solution to the positive real control problem for linear time-invariant systems," IEEE Transactions on Automatic Control, vol. 39, no. 10, pp. 2034-2046, Oct 1994.

[26] Z. Song, A. Lanzon, S. Patra, and I. R. Petersen, "Towards controller synthesis for systems with negative imaginary frequency response," IEEE Transactions on Automatic Control, vol. 55, no. 6, pp. 15061511, Jun 2010 